\title{
Heterogeneous Patterns of Acid Phosphatases Containing Low-Molecular-Mass Polypeptides in Members of the Family Enterobacteriaceae
}

\author{
MARIA CRISTINA THALLER, ${ }^{1}$ FRANCESCA BERLUTTI, ${ }^{1}$ SERENA SCHIPPA, ${ }^{1}$ PATRIZIA IORI, ${ }^{1}$ \\ CLAUDIO PASSARIELLO ${ }^{1}$ AND GIAN MARIA ROSSOLINI ${ }^{2 *}$ \\ Istituto di Microbiologia, Università "La Sapienza," 00185, Rome, ${ }^{1}$ and Dipartimento di Biologia \\ Molecolare, Sezione di Microbiologia, Università di Siena, 53100 Siena, ${ }^{2}$ Italy
}

\begin{abstract}
We investigated expression of acid phosphatases containing low-molecular-mass ( 25 to 27-kDa) polypeptides (Lmmp-APs) similar to those described previously for Salmonella enterica serovar typhimurium and Morganella morganii in strains that were representatives of 43 different enterobacterial species by using a zymogram technique developed for detection of Lmmp-AP activities and for analysis of some of the properties of these enzymes. Under conditions that were suitable for detection of the previously described Lmmp-APs, production of similar enzymes appeared to be widespread but not universal among enteric bacteria, and heterogeneous patterns of expression were found among strains belonging to different genera and, in some cases, among strains belonging to different species of the same genus. We found that class A Lmmp-APs (i.e., Lmmp-Aps similar to the Morganella morganii PhoC and Salmonella enterica serovar typhimurium PhoN acid phosphatases) were also expressed in Cedecea spp., Enterobacter aerogenes, Hafnia alvei, Klebsiella spp., Providencia stuartii, Serratia plymuthica, and Yokenella regensburgei strains and that class B Lmmp-APs (i.e., Lmmp-APs similar to the Morganella morganii NapA and Salmonella enterica serovar typhimurium NapII acid phosphatases) were also expressed in strains of Citrobacter spp., Escherichia coli, Escherichia fergusonii, Hafnia alvei, Proteus mirabilis, Providencia spp., Salmonella enterica serovar typhi, Shigella dysenteriae, and Shigella flexneri. No Lmmp-AP activity was detected in strains of Enterobacter spp. other than Enterobacter aerogenes, Escherichia hermanii, Kluyvera ascorbata, Leclercia adecarboxylata, Leminorella grimontii, Moellerella wisconsensis, Proteus vulgaris, Proteus penneri, Serratia spp. other than Serratia plymuthica, and Yersinia spp. Because of the heterogeneous patterns of expression of Lmmp-APs, analysis of these enzymes could be useful for evolutionary studies of the enterobacterial genome and for precise phylogenetic positioning of enteric bacteria.
\end{abstract}

Phosphatase activity occurs in all members of the family Enterobacteriaceae (18), but different patterns of phosphatase activity have been found in strains belonging to this family $(2$, $3,10,12,13,19)$. For instance, it is known that a phosphaterepressible alkaline phosphatase is widespread but not universally present in enterobacterial species $(2,13,19)$. It is also known that the levels of cyclic phosphodiesterase activity and 5 '-nucleotidase (UDP-sugar hydrolase) activity can be markedly different in different species (10) and that two different types of $5^{\prime}$-nucleotidases are produced by Escherichia coli and Salmonella spp. as a consequence of alternative silencing events in the genes that encode these enzymes (3). Moreover, only some species (Morganella morganii and Providencia stuartii) produce high levels of phosphate-irrepressible acid phosphatase activity (HPAP phenotype), while other species produce only intermediate or low levels of acid phosphatase activity when $P_{i}$ is freely available in the culture medium (12, 13).

However, knowledge concerning enterobacterial phosphatases is incomplete, and the data are somewhat confusing since most of the available data concern phosphatase activity as a whole, while individual enzymatic activities have been studied in detail only in Escherichia coli and, to a lesser extent, in a few other species. Moreover, different methods have been used to evaluate phosphatase activities in different studies, thus rendering comparisons difficult and conclusions questionable.

\footnotetext{
* Corresponding author. Mailing address: Dipartimento di Biologia Molecolare, Sezione di Microbiologia, Università di Siena, Via Laterina, 8, 53100 Siena, Italy. Phone: 39-577-280903. Fax: 39-577-42011.
}

For the reasons stated above, more knowledge concerning the enterobacterial phosphatases and their diversity could be relevant not only in the field of microbial physiology and enzymology, but also for taxonomic, phylogenetic, and diagnostic purposes.

Among the different phosphatases found in members of the Enterobacteriaceae, a potentially interesting but still poorly characterized group is represented by the nonspecific periplasmic acid phosphatases containing low-molecular-mass (around 25- to $27-\mathrm{kDa}$ ) polypeptides (Lmmp-APs). Similar enzymes have far been described in Salmonella enterica serovar typhimurium (4, 6, 7, 23-25), Morganella morganii (21, 22), Providencia stuartii (15), and Escherichia coli (16), but only the Salmonella and the Morganella enzymes have been purified and characterized in detail. At least two different families of these enzymes can be defined on the basis of structural and functional properties. One family includes the PhoN enzyme of Salmonella enterica serovar typhimurium $(4,7,24,25)$ and the structurally and functionally homologous enzymes of Morganella morganii (21) and Providencia stuartii (15), which have been designated the class A bacterial acid phosphatases (21). The other family includes the NapII enzyme of Salmonella enterica serovar typhimurium $(23,24)$ and the NapA enzyme of Morganella morganii (22), which have been designated the class $\mathrm{B}$ bacterial acid phosphatases (22). We have also recently reported that Escherichia coli can produce a nonspecific periplasmic Lmmp-AP containing a $27-\mathrm{kDa}$ polypeptide component (16), but it is not known yet whether this enzyme belongs to one of the two families described above.

In this study we investigated the production of similar enzymes in strains that represented several different enterobac- 
terial species by using a zymogram technique which was able to resolve different activities and to provide, at the same time, information concerning the size of the polypeptide component, the optimum $\mathrm{pH}$ value, and activity with different substrates. Our results showed that expression of Lmmp-APs with properties similar to those described previously is widespread although not universal among members of the Enterobacteriaceae and that heterogeneous patterns of expression are found among strains belonging to different genera and, in some cases, also among strains belonging to different species of the same genus.

\section{MATERIALS AND METHODS}

Bacterial strains. The strains used in this study are listed in Table 1. All strains were stored at $-80^{\circ} \mathrm{C}$ in brain heart infusion broth (Difco Laboratories, Detroit, Mich.) supplemented with $20 \%$ (vol/vol) glycerol until they were used.

Culture media and conditions. Terrific broth (TB) (17) was composed of $1.2 \%$ (wt/vol) tryptone (Difco), 2.4\% (wt/vol) yeast extract (Difco), $90 \mathrm{mM}$ sodium phosphate buffer ( $\mathrm{pH} 7.2$ ), and $0.4 \%$ (vol/vol) glycerol. TB was used as the growth medium for analysis of Lmmp-APs since it supported the growth of all of the strains tested and was suitable for detection of all of the previously described Lmmp-APs. Cells were always grown aerobically at $37^{\circ} \mathrm{C}$.

Zymogram techniques. Phosphatase activities were analyzed in whole-cell protein preparations by a zymogram technique. To prepare whole-cell proteins, bacteria were washed in normal saline, resuspended in normal saline at an optical density at $600 \mathrm{~nm}$ of 10 , and disrupted by sonication. Cell debris was then removed by centrifugation at $10,000 \times g$ for $10 \mathrm{~min}$ at $4^{\circ} \mathrm{C}$. For zymograms, the protein preparations were subjected to sodium dodecyl sulfate (SDS)-polyacrylamide gel electrophoresis (PAGE) under completely or partially denaturing conditions. Samples used for SDS-PAGE under completely denaturing conditions were boiled for $5 \mathrm{~min}$ in Laemmli's sample buffer (8) before loading, while samples used for SDS-PAGE under partially denaturing conditions were mixed with Laemmli's sample buffer at $0^{\circ} \mathrm{C}$ and loaded without any preliminary exposure to heat (23). SDS-PAGE was always performed with a minigel apparatus (model SE200; Hoefer Scientific Instruments, San Francisco, Calif.) by using an acrylamide concentration of $15 \%$ in the resolving gel. After electrophoresis, the gels were incubated for $3 \mathrm{~h}$ at $37^{\circ} \mathrm{C}$ in several changes of renaturation buffer to obtain renaturation of enzymes. The renaturation buffer contained $10 \mathrm{mM}$ Tris$\mathrm{HCl}(\mathrm{pH} 7), 2 \mathrm{mM} \mathrm{MgSO}, 0.05 \mathrm{mM} \mathrm{ZnCl}_{2}$, and $1 \%$ (vol/vol) deionized Triton $\mathrm{X}-100$. After renaturation, the gels were equilibrated for $1 \mathrm{~h}$ in several changes of either $100 \mathrm{mM}$ sodium acetate buffer (pH 5.5) or $100 \mathrm{mM}$ glycine- $\mathrm{NaOH}$ buffer ( $\mathrm{pH} 9.5)$ and then processed for detection of phosphatase activities. Two different detection systems were used. In one system (the MG system) (16), after the equilibration step the gel was incubated at $37^{\circ} \mathrm{C}$ for $14 \mathrm{~h}$ in equilibration buffer containing $4 \mathrm{mM}$ phenolphthalein diphosphate (PDP) (tetrasodium salt; Sigma Chemical Co., St. Louis, Mo.) and $0.05 \mathrm{~g}$ of the stain methyl green (Sigma) per liter. In this system phosphatase activities were indicated by the presence of bands composed of a green precipitate (at the acidic $\mathrm{pH}$ value) or a white precipitate (at the alkaline $\mathrm{pH}$ value). In the other system (the 5-bromo-4-chloro3-indolylphosphate [BCIP] detection system), after the equilibration step the gel was incubated at $37^{\circ} \mathrm{C}$ for $14 \mathrm{~h}$ in equilibration buffer containing $0.25 \mathrm{mM}$-BCIP (Sigma). In this system phosphatase activities were indicated by the presence of bands composed of a blue precipitate. It should be noted that in all cases, the phosphatase activity bands were located at a migration distance corresponding to the $M_{\mathrm{r}}$ of the polypeptide component of the enzyme. Inhibition assays were performed by adding an inhibitor ( $20 \mathrm{mM}$ [final concentration] EDTA or $1 \mathrm{mM}$ [final concentration] fluoride) to the equilibration buffer.

\section{RESULTS}

Zymogram detection and identification of enterobacterial Lmmp-APs. Although it has been reported that Escherichia coli produces an Lmmp-AP (16) and that a class A Lmmp-AP has been cloned from Providencia stuartii (15), the only enterobacterial Lmmp-APs that have been purified and characterized in detail previously are those of Salmonella enterica serovar typhimurium (4, 7, 23-25) and Morganella morganii (21, 22). These Lmmp-APs include two class A enzymes (PhoN in Salmonella strains and PhoC in Morganella strains) and two class B enzymes (NapII in Salmonella strains and NapA in Morganella strains). In this study, we first developed a zymogram technique which could be used to detect and identify Lmmp-AP activities (see Materials and Methods for details).

Both types of Lmmp-APs could be detected in early-station- ary-phase cells of Salmonella enterica serovar typhimurium ATCC 15277 (= LT2) or Morganella morganii ATCC $25830^{\mathrm{T}}$ $(\mathrm{T}=$ type strain) grown in $\mathrm{TB}$ with the zymogram technique when we developed the gels at an acidic $\mathrm{pH}$ value and used PDP as the substrate and the MG detection system (Fig. 1a, gel D). Because their polypeptide sizes were similar, the two Lmmp-APs of each species migrated close to each other. In fact, the class A phosphatase appeared to be only slightly larger and slightly smaller than the class B enzyme in Salmonella and Morganella preparations, respectively (Fig. 1a, gel D). Clearcut resolution of the two activities could be achieved only if a suitable amount of sample was loaded onto the gel (75 to 100 $\mu \mathrm{g}$ of total protein was found to be optimal; larger amounts resulted in large bands of activity which could actually overlap with each other), and the two polypeptides were properly resolved by electrophoresis (an acrylamide concentration in the resolving gel of $15 \%$ was found to be optimal when we used a minigel apparatus). The two classes of enzymes could then be distinguished from each other on the basis of the following properties. Unlike the class A enzymes, the class B Lmmp-APs migrated mostly as higher- $M_{\mathrm{r}}$ polymers if cell proteins were subjected to SDS-PAGE under partially denaturing conditions (Fig. 1a, gel PD), and the activity of class B enzymes was not inhibited by the presence of $0.01 \%$ (wt/vol) SDS (Fig. 1b). On the other hand, unlike the class B enzymes, the class A LmmpAPs were active on BCIP, and their activity on PDP or BCIP was not inhibited by the presence of $20 \mathrm{mM}$ EDTA (Fig. 1c).

It should be noted that, notwithstanding their overall similarity, the class A enzymes of Salmonella enterica serovar typhimurium and Morganella morganii also differ in some respects and have different phylogenetic histories and therefore probably are members of two different subclasses. In fact, unlike the Morganella homolog, which has a homotetrameric quaternary structure, is resistant to fluoride, and is encoded by a gene that apparently was acquired early in the lineage (21), the Salmonella PhoN enzyme has a homodimeric quaternary structure, is inhibited by fluoride, and is encoded by a gene that apparently was derived from a recent horizontal transfer $(4,7$, 24). Moreover, the Salmonella PhoN polypeptide component is also slightly larger than the polypeptide component of the Morganella PhoC phosphatase $(4,21)$. Some of these distinctive characteristics could be exploited in zymograms to distinguish these two types of class A enzymes (Fig. 1). We propose that the enzyme groups which contain Morganella and Salmonella class A enzymes should be designated classes A1 and A2, respectively.

It should also be noted that under the experimental conditions described above, a band of phosphatase activity was also often detected at an $M_{\mathrm{r}}$ of approximately $44 \mathrm{kDa}$ (Fig. 1). This band corresponds to an acid hexose-phosphatase (20) which in Salmonella enterica serovar typhimurium has been described previously $(7,24,25)$ and is apparently homologous to the Escherichia coli Agp enzyme (14).

On the basis of the data described above, we investigated the presence of similar Lmmp-APs in strains that represent several different enterobacterial species by using the zymogram technique.

Screening for Lmmp-APs in members of the family Enterobacteriaceae. Representative strains of various enterobacterial species were assayed for the presence of Lmmp-APs by the zymogram technique described above. The screening assays were performed with early-stationary-phase cells grown in TB by using SDS-PAGE under completely denaturing conditions, PDP as the substrate, and the MG detection system (i.e., conditions known to be suitable for detecting previously described Lmmp-APs [see above]). 
TABLE 1. Lmmp-APs detected by the zymogram technique in representatives of several enterobacterial species ${ }^{a}$

\begin{tabular}{|c|c|c|}
\hline Strain $^{b}$ & Presence of $\operatorname{LmmAP}(\mathrm{s})$ & $M_{\mathrm{r}}(\mathrm{s})\left(10^{3}\right)$ \\
\hline $\begin{array}{l}\text { Cedecea davisae CIP } 8034^{\mathrm{T}} \\
\text { Cedecea neteri ATCC } 33855^{\mathrm{T}}\end{array}$ & $\begin{array}{l}+ \\
+\end{array}$ & $\begin{array}{l}25 \\
25\end{array}$ \\
\hline $\begin{array}{l}\text { Citrobacter amalonaticus ATCC } 25405^{\mathrm{T}} \\
\text { Citrobacter koseri CIP } 7214^{c} \\
\text { Citrobacter freundii ATCC } 8090^{\mathrm{T}}\end{array}$ & $\begin{array}{l}+ \\
+ \\
+\end{array}$ & $\begin{array}{l}27 \\
27 \\
27\end{array}$ \\
\hline $\begin{array}{l}\text { Enterobacter aerogenes CIP } 6086^{\mathrm{T}} \\
\text { Enterobacter agglomerans ATCC } 29904 \\
\text { Enterobacter amnigenus ATCC } 33072^{\mathrm{T}} \\
\text { Enterobacter cloacae CIP } 6085^{\mathrm{T}} \\
\text { Enterobacter sakazakii ATCC } 29544^{\mathrm{T}} \\
\text { Enterobacter taylorae ATCC } 35317^{\mathrm{T}}\end{array}$ & $\begin{array}{l}+ \\
- \\
- \\
- \\
-\end{array}$ & 25 \\
\hline $\begin{array}{l}\text { Escherichia coli MG1655 (= CGSC 6300) } \\
\text { Escherichia fergusonii ATCC } 35469^{\mathrm{T}} \\
\text { Escherichia hermanii ATCC } 33650^{\mathrm{T}}\end{array}$ & $\begin{array}{l}+ \\
+ \\
+\end{array}$ & $\begin{array}{l}27 \\
27\end{array}$ \\
\hline Hafnia alvei ATCC 29926 & + & 25,27 \\
\hline $\begin{array}{l}\text { Klebsiella oxytoca CIP } 666 \\
\text { Klebsiella planticola CIP } 8136^{d} \\
\text { Klebsiella pneumoniae CIP } 52144 \\
\text { Klebsiella terrigena CIP } 8007^{\mathrm{T}}\end{array}$ & $\begin{array}{l}+ \\
+ \\
+ \\
+\end{array}$ & $\begin{array}{l}25 \\
25 \\
25 \\
25\end{array}$ \\
\hline Kluyvera ascorbata ATCC 33434 & - & \\
\hline Leclercia adecarboxylata CIP 100921 & - & \\
\hline Leminorella grimontii ATCC $33999^{\mathrm{T}}$ & - & \\
\hline Moellerella wisconsensis ATCC $35017^{\mathrm{T}}$ & - & \\
\hline Morganella morganii ATCC $25830^{\mathrm{T}}$ & + & $25,27^{e}$ \\
\hline $\begin{array}{l}\text { Proteus mirabilis ATCC } 29906^{\mathrm{T}} \\
\text { Proteus penneri ATCC } 33519^{\mathrm{T}} \\
\text { Proteus vulgaris ATCC } 8427\end{array}$ & $\begin{array}{l}+ \\
- \\
-\end{array}$ & 27 \\
\hline $\begin{array}{l}\text { Providencia alcalifaciens CIP } 5862 \\
\text { Providencia rettgeri ATCC } 29944^{\mathrm{T}} \\
\text { Providencia rustigianii ATCC } 33673^{\mathrm{T}} \\
\text { Providencia stuartii ATCC } 29914^{\mathrm{T}}\end{array}$ & $\begin{array}{l}+ \\
+ \\
+ \\
+\end{array}$ & $\begin{array}{l}27 \\
27 \\
27 \\
25,27^{e}\end{array}$ \\
\hline $\begin{array}{l}\text { Salmonella enterica serovar typhi ATCC } 19430^{(\mathrm{T})}(=\mathrm{Ty} 2) \\
\text { Salmonella enterica } \text { serovar typhimurium ATCC } 15277(=\mathrm{LT} 2)\end{array}$ & $\begin{array}{l}+ \\
+\end{array}$ & $\begin{array}{l}27 \\
27,28\end{array}$ \\
\hline $\begin{array}{l}\text { Serratia fonticola CIP } 7864^{\mathrm{T}} \\
\text { Serratia liquefaciens CIP } 674 \\
\text { Serratia marcescens CIP } 6755 \\
\text { Serratia odorifera CIP } 7901^{\mathrm{T}} \\
\text { Serratia plymuthica CIP } 7712\end{array}$ & $\begin{array}{l}- \\
- \\
- \\
+\end{array}$ & 28 \\
\hline $\begin{array}{l}\text { Shigella dysenteriae NCTC } 4837^{\mathrm{T}} \\
\text { Shigella flexneri CIP } 8248\end{array}$ & $\begin{array}{l}+ \\
+\end{array}$ & $\begin{array}{l}27 \\
27\end{array}$ \\
\hline $\begin{array}{l}\text { Yersinia enterocolitica CIP } 8027^{\mathrm{T}} \\
\text { Yersinia pseudotuberculosis } \mathrm{Yss}^{\mathrm{T}} 133^{f} \\
\text { Yersinia kristensenii CIP } 8030^{\mathrm{T}}\end{array}$ & $\begin{array}{l}- \\
-\end{array}$ & \\
\hline Yokenella regensburgei ATCC $35313^{g}$ & + & 25 \\
\hline
\end{tabular}

${ }^{a}$ In these experiments zymograms were prepared after electrophoresis under completely denaturing conditions and were developed by using PDP as the substrate and the MG system for detection (see Materials and Methods for further details concerning the zymogram technique). For each strain, approximately $100 \mu g$ of total protein prepared from early-stationary-phase cells grown in TB was loaded onto the gel. Results were reproducible in duplicate experiments.

${ }^{b}$ CIP, Collection of the Institut Pasteur; ATCC, American Type Culture Collection.

${ }^{c}$ Obtained as the type strain of Levinea malonatica.

${ }^{d}$ Obtained as the type strain of Klebsiella trevisanii.

$e^{e}$ The $25-\mathrm{kDa}$ bands of activity in these strains were larger and more intense than all of the other bands detected.

$f$ Clinical isolate obtained from the culture collection of the Italian National Institute of Health, Rome.

${ }^{g}$ Obtained as the type strain of Koserella trabulsii. 
$M_{r}(k D a)$

D
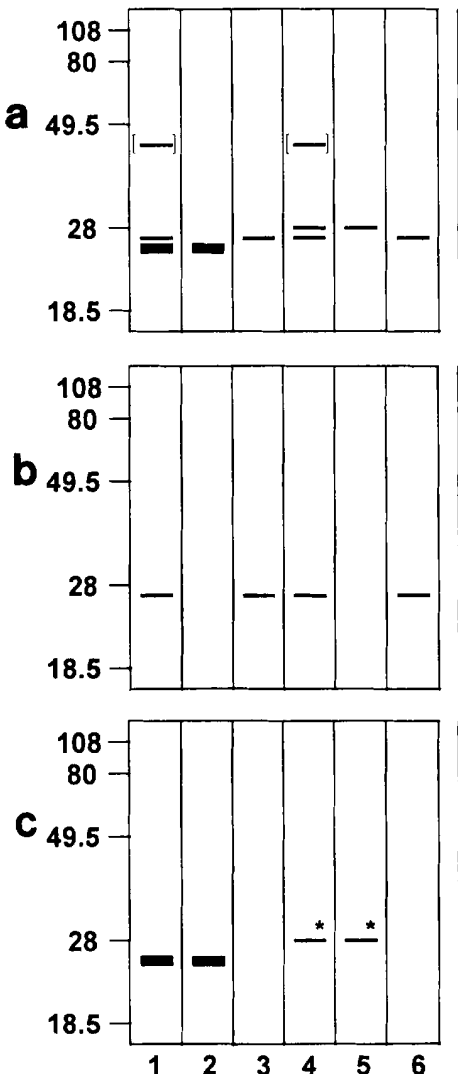

PD
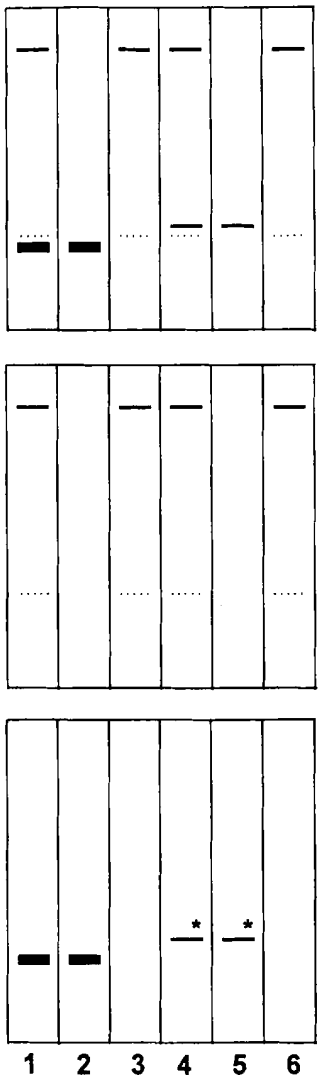

Lmmp-AP

Class B (partially denatured)

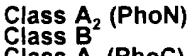

Class $\mathrm{A}_{1}$ (PhoC)

Class B (partially denatured)

Class B

Class $\mathrm{A}_{2}$ (PhoN)

Class $A_{1}$ (PhoC)

FIG. 1. Schematic representation of zymogram detection of the Lmmp-APs of Morganella morganii ATCC $25830^{\mathrm{T}}$ and of Salmonella enterica serovar typhimurium ATCC 15277 (= LT2). Gels D, and PD, gels after SDS-PAGE performed under completely and partially denaturing conditions, respectively (see Materials and Methods for details concerning the electrophoretic conditions used). (a) Results obtained when the gel was equilibrated at pH 5.5 and developed by using PDP as the substrate and the MG detection system. ( $b$ and $c$ ) Results obtained when the gel was equilibrated and developed as described above, but with $0.01 \%$ (wt/vol) SDS and $20 \mathrm{mM}$ EDTA, respectively, added starting from the equilibration step. When the gel was equilibrated at $\mathrm{pH} 5.5$ and developed by using the BCIP detection system, the activity pattern was identical to the pattern shown in panel $\mathrm{c}$. The asterisks indicate bands that did not appear when $1 \mathrm{mM}$ fluoride was added after the equilibration step. Lanes 1, Morganella morganii ATCC $25830^{\mathrm{T}}$ whole-cell proteins; lane 2, purified Morganella morganii PhoC acid phosphatase; lane 3, purified Morganella morganii NapA acid phosphatase; lane 4, Salmonella enterica serovar typhimurium ATCC 15277 (= LT2) whole-cell proteins; lane 5, purified Salmonella enterica serovar typhimurium PhoN acid phosphatase; lane 6, purified Salmonella enterica serovar typhimurium NapII acid phosphatase. In lanes in which the results obtained with whole-cell proteins are shown (lanes 1 and 4) the band thickness matches with the actual proportions of the proteins. The bands shown as dotted lines represent the small amount of class B enzyme which was completely denatured and migrated to an $M_{\mathrm{r}}$ of $27 \mathrm{kDa}$ when electrophoresis was performed under partially denaturing conditions. The bands in brackets, located at an $M_{\mathrm{r}}$ of approximately $44 \mathrm{kDa}$, represent a hexose phosphatase which was often detected under the experimental conditions used (20). Purified Morganella morganii Lmmp-APs were obtained from our laboratories $(21,22)$. Purified Salmonella enterica serovar typhimurium phosphatases were obtained from Salmonella enterica serovar typhimurium ATCC 15277 (= LT2) as previously described (23, 24).

The results of this analysis showed that in addition to Morganella morganii ATCC $25830^{\mathrm{T}}$ and Salmonella enterica serovar typhimurium ATCC 15277 (= LT2), as well as Providencia stuartii ATCC $29914^{\mathrm{T}}$ and Escherichia coli MG1655 (for which production of Lmmp-APs has been reported previously [15, 16]), strains belonging to several other enterobacterial species produced Lmmp-AP activities (Table 1). In particular, Hafnia alvei ATCC 29926 and Providencia stuartii ATCC $29914^{\mathrm{T}}$ produced two clearly distinguishable bands of activity, like Morganella morganii ATCC $25830^{\mathrm{T}}$ and Salmonella enterica serovar typhimurium ATCC 15277 (= LT2), while Cedecea davisae CIP $8034^{\mathrm{T}}$, Cedecea neteri ATCC $33855^{\mathrm{T}}$, Citrobacter amalonaticus ATCC $25405^{\mathrm{T}}$, Citrobacter koseri CIP 7214, Citrobacter freundii ATCC $8090^{\mathrm{T}}$, Enterobacter aerogenes CIP $6086^{\mathrm{T}}$, Escherichia coli MG1655, Escherichia fergusonii ATCC $35469^{\mathrm{T}}$, Klebsiella oxytoca CIP 666, Klebsiella planticola CIP 8136, Klebsiella pneumoniae CIP 52144, Klebsiella terrigena CIP $8007^{\mathrm{T}}$, Proteus mirabilis ATCC $29906^{\mathrm{T}}$, Providencia alcalifaciens CIP 5862 , Providencia rettgeri ATCC $29944^{\mathrm{T}}$, Providencia rustigianii
ATCC $33673^{\mathrm{T}}$, Salmonella enterica serovar typhi ATCC $19430^{(\mathrm{T})}$, Serratia plymuthica CIP 7712, Shigella dysenteriae NCTC $4837^{\mathrm{T}}$, Shigella flexneri CIP 8248, and Yokenella regensburgei ATCC 35313 produced only one band of Lmmp-AP activity. However, the possibility that more than one band of activity that comigrated at the same distance was present could not be eliminated at this stage for these strains. No Lmmp-APs were detected in Enterobacter agglomerans ATCC 29904, Enterobacter amnigenus ATCC $33072^{\mathrm{T}}$, Enterobacter cloacae CIP $6085^{\mathrm{T}}$, Enterobacter sakazakii ATCC $29544^{\mathrm{T}}$, Enterobacter taylorae ATCC $35317^{\mathrm{T}}$, Escherichia hermanii ATCC $33650^{\mathrm{T}}$, Kluyvera ascorbata ATCC 33434, Leclercia adecarboxylata CIP 100921, Leminorella grimontii ATCC $33999^{\mathrm{T}}$, Moellerella wisconsensis ATCC $35017^{\mathrm{T}}$, Proteus penneri ATCC $33519^{\mathrm{T}}$, Proteus vulgaris ATCC 8427, Serratia fonticola CIP $7864^{\mathrm{T}}$, Serratia liquefaciens CIP 674, Serratia marcescens CIP 6755, Serratia odorifera CIP $7901^{\mathrm{T}}$, Yersinia enterocolitica CIP $8027^{\mathrm{T}}$, Yersinia pseudotuberculosis Yss133, and Yersinia kristensenii CIP $8030^{\mathrm{T}}$. All of the Lmmp-AP activities detected at the acidic $\mathrm{pH}$ 
value were inactive or exhibited greatly reduced activity at the alkaline $\mathrm{pH}$ value (data not shown), and phosphatases containing low-molecular-mass polypeptides that were active only at alkaline $\mathrm{pH}$ values were never observed. The intensities of bands of activity were somewhat variable. The larger and more intense bands were the 25-kDa bands of activity observed with Morganella morganii ATCC $25830^{\mathrm{T}}$ and Providencia stuartii ATCC $29914^{\text {T }}$ (Table 1).

As observed with Morganella morganii ATCC $25830^{\mathrm{T}}$ and Salmonella enterica serovar typhimurium ATCC 15277 (= LT2) (see above), under the experimental conditions which we used activities of other acid phosphatases containing larger (always $>40-\mathrm{kDa}$ ) polypeptides were sometimes detected (data not shown). Such activities were not further characterized in this study.

Characterization of the Lmmp-APs detected in different species. All of the Lmmp-AP activities detected in strains of species other than Salmonella enterica serovar typhimurium and Morganella morganii were analyzed by performing additional tests, including electrophoresis under partially denaturing conditions and tests for inhibition by SDS, inhibition by EDTA, activity on BCIP, and, for putative class A activities, inhibition by fluoride.

The results of this analysis showed that the Lmmp-APs of Cedecea davisae CIP $8034^{\mathrm{T}}$, Cedecea neteri ATCC $33855^{\mathrm{T}}$, Enterobacter aerogenes CIP $6086^{\mathrm{T}}$, Klebsiella oxytoca CIP 666 , Klebsiella planticola CIP 8136, Klebsiella pneumoniae CIP 52144, Klebsiella terrigena CIP $8007^{\mathrm{T}}$, Serratia plymuthica CIP 7712, and Yokenella regensburgei ATCC 35313 were inhibited by SDS, were resistant to EDTA, were active on BCIP, and did not migrate as larger polymers under partially denaturing conditions and thus apparently were class A enzymes. No class B or other Lmmp-AP activities were detected in these strains (Table 2). On the other hand, the Lmmp-APs of Citrobacter amalonaticus ATCC $25405^{\mathrm{T}}$, Citrobacter koseri CIP 7214, Citrobacter freundii ATCC $8090^{\mathrm{T}}$, Escherichia coli MG1655, Escherichia fergusonii ATCC $35469^{\mathrm{T}}$, Proteus mirabilis ATCC $29906^{\mathrm{T}}$, Providencia alcalifaciens CIP 5862, Providencia rettgeri ATCC $29944^{\mathrm{T}}$, Providencia rustigianii ATCC $33673^{\mathrm{T}}$, Salmonella enterica serovar typhi ATCC $19430^{(\mathrm{T})}$, Shigella dysenteriae NCTC $4837^{\mathrm{T}}$, and Shigella flexneri CIP 8248 were resistant to SDS, were inhibited by EDTA, were inactive on BCIP, and migrated as larger polymers under partial denaturing conditions and thus apparently were class B enzymes. No class A or other Lmmp-AP activities were detected in these strains (Table 2). The two Lmmp-APs found in Hafnia alvei ATCC 29926 and in Providencia stuartii ATCC $29914^{\mathrm{T}}$ behaved like a class A enzyme (the smaller enzyme) and a class B enzyme (the larger enzyme) (Table 2).

Of the class A enzymes found in this study, only the class A enzyme of Serratia plymuthica CIP 7712 was inhibited by fluoride and contained a slightly larger polypeptide component (like the Salmonella enterica serovar typhimurium class A enzyme) and was therefore classified as a class A2 enzyme, while all of the other class A enzymes were resistant to fluoride and contained a slightly smaller polypeptide component (like the Morganella morganii class A enzyme) and were therefore classified as class A1 enzymes (Table 2).

\section{DISCUSSION}

So far, production of Lmmp-APs has been reported only for some enterobacterial species. There are at least two different families of such enzymes, which differ in their structural and functional properties; these enzymes have been designated the class A and class B bacterial acid phosphatases $(21,22)$.
Using the simple zymogram procedure described in this paper, which was suitable for detecting and identifying the known Lmmp-APs, we screened several representatives of the family Enterobacteriaceae for the presence of similar Lmmp-APs, and our analysis showed that production of similar enzymes is actually widespread, although not universal, among members of this family. All of the Lmmp-APs which we detected were apparently either class A or class B enzymes. However, the presence of additional types of Lmmp-APs cannot be definitely ruled out since the detection conditions which we used may not be suitable for detection of different enzymes.

The pattern of Lmmp-AP production was rather heterogeneous. Some strains apparently were not able to express any Lmmp-AP activity, while most strains expressed at least one Lmmp-AP activity (either class A or class B) and a few strains simultaneously produced both a class A enzyme and a class B enzyme. These different phenotypes could be related to differences in regulation of the Lmmp-AP-encoding genes (so that the assay conditions which we used may not have elicited Lmmp-AP production in some cases) or to a true inability to produce Lmmp-AP based on genotype. We are currently investigating these possibilities by examining expression of class $A$ and class B activities under different growth conditions and by investigating the presence of sequences homologous to the known class $\mathrm{A}$ and class $\mathrm{B}$ phosphatase-encoding genes in strains that apparently are not able to produce the enzymes. In any case, the heterogeneity of Lmmp-APs in enteric bacteria, like the heterogeneity found for other phosphatases, indicates that the Lmmp-AP genes and/or their regulatory elements were involved in changes which occurred in the enterobacterial genome after different species diverged. For this reason, analysis of similar enzymes could be useful for evolutionary studies of enterobacterial genomes and for precise phylogenetic positioning of enteric bacteria, for which rRNA sequencing alone is not sensitive enough $(1,9)$. It should be noted that production of class B Lmmp-APs by Escherichia coli MG1655 and Escherichia fergusonii ATCC $35469^{\mathrm{T}}$ but not by Escherichia hermanii ATCC $33650^{\mathrm{T}}$ is fully consistent with the hypothesis that only Escherichia coli and Escherichia fergusonii should be placed in genus Escherichia, while Escherichia hermanii, Escherichia blattae, and Escherichia vulneris should be placed in different genera (9), and that production of class A1 enzymes by strains belonging to different Klebsiella species and by Enterobacter aerogenes CIP $6086^{\mathrm{T}}$ but not by strains belonging to other Enterobacter species is consistent with the hypothesis that Enterobacter aerogenes is more closely related to klebsiellae than to other enterobacters and should be reclassified in the genus Klebsiella $(1,5)$.

Production of class B Lmmp-APs appeared to be quite widespread among enteric bacteria. In fact, in addition to the Salmonella enterica serovar typhimurium and Morganella morganii class B enzymes, class B enzymes were also found in strains belonging to following species: Citrobacter amalonaticus, Citrobacter koseri, Citrobacter freundii, Escherichia coli, Escherichia fergusonii, Proteus mirabilis, Providencia alcalifaciens, Providencia rettgeri, Providencia rustigianii, Providencia stuartii, Salmonella enterica serovar typhi, Shigella dysenteriae, and Shigella flexneri. The class B Lmmp-AP detected in Escherichia coli MG1655 apparently corresponded to the p27 activity previously described in the same strain (16) and therefore the p27 activity can be classified as a class B enzyme. Considering our current knowledge of enterobacterial phylogenesis $(1,11)$ and the distribution of class $\mathrm{B}$ acid phosphatases, it is possible to speculate that an ancestral class $B$ gene was present in the enterobacterial ancestor or was acquired early in the lineage and that during subsequent evolution of the family this gene 
TABLE 2. Characterization of the Lmmp-APs detected in the strains which we used ${ }^{a}$

\begin{tabular}{|c|c|c|c|c|c|c|c|c|c|c|}
\hline \multirow[b]{3}{*}{ Strain } & \multicolumn{9}{|c|}{ Lmmp-AP activities after SDS-PAGE under: } & \multirow[b]{3}{*}{ 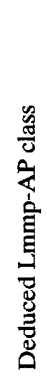 } \\
\hline & \multicolumn{5}{|c|}{ Denaturing conditions } & \multicolumn{4}{|c|}{ Partially denaturing conditions } & \\
\hline & $\underbrace{\stackrel{9}{巳}}_{2}$ & 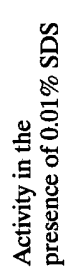 & 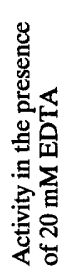 & 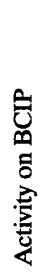 & 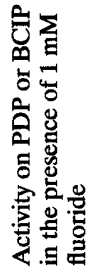 & $\underbrace{\stackrel{0}{0}}_{=}$ & 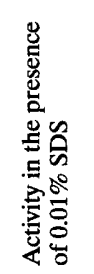 & 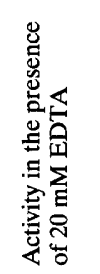 & 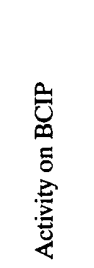 & \\
\hline Cedecea davisae CIP $8034^{\mathrm{T}}$ & 25 & - & + & + & + & 25 & - & + & + & A1 \\
\hline Cedecea neteri ATCC $33855^{\mathrm{T}}$ & 25 & - & + & + & + & 25 & - & + & + & A1 \\
\hline Citrobacter amalonaticus ATCC $25405^{\mathrm{T}}$ & 27 & + & - & - & $\mathrm{ND}^{b}$ & $100(27)^{c}$ & $+(+)$ & $-(-)$ & $-(-)$ & B \\
\hline Citrobacter koseri CIP 7214 & 27 & + & - & - & ND & $100(27)$ & $+(+)$ & $-(-)$ & $-(-)$ & B \\
\hline Citrobacter freundii ATCC $8090^{\mathrm{T}}$ & 27 & + & - & - & ND & $100(27)$ & $+(+)$ & $-(-)$ & $-(-)$ & B \\
\hline Enterobacter aerogenes CIP $6086^{\mathrm{T}}$ & 25 & - & + & + & + & 25 & - & + & + & A1 \\
\hline Escherichia coli MG1655 & 27 & + & - & - & ND & $100(27)$ & $+(+)$ & $-(-)$ & $-(-)$ & B \\
\hline Escherichia fergusonii ATCC $35469^{\mathrm{T}}$ & 27 & + & - & - & ND & $100(27)$ & $+(+)$ & $-(-)$ & $-(-)$ & B \\
\hline Hafnia alvei ATCC 29926 & 25 & - & + & + & + & 25 & - & + & + & A1 \\
\hline & 27 & + & - & - & ND & $100(27)$ & $+(+)$ & $-(-)$ & $-(-)$ & B \\
\hline Klebsiella oxytoca CIP 666 & 25 & - & + & + & + & 25 & - & + & + & A1 \\
\hline Klebsiella planticola CIP 8136 & 25 & - & + & + & + & 25 & - & + & + & A1 \\
\hline Klebsiella pneumoniae CIP 52144 & 25 & - & + & + & + & 25 & - & + & + & A1 \\
\hline Klebsiella terrigena $\mathrm{CIP} 8007^{\mathrm{T}}$ & 25 & - & + & + & + & 25 & - & + & + & A1 \\
\hline Morganella morganii ATCC $25830^{\mathrm{T}}$ & 25 & - & + & + & + & 25 & - & + & + & A1 \\
\hline & 27 & + & - & - & ND & $100(27)$ & $+(+)$ & $-(-)$ & $-(-)$ & B \\
\hline Proteus mirabilis ATCC $29906^{\mathrm{T}}$ & 27 & + & - & - & ND & $100(27)$ & $+(+)$ & $-(-)$ & $-(-)$ & B \\
\hline Providencia alcalifaciens CIP 5862 & 27 & + & - & - & ND & $100(27)$ & $+(+)$ & $-(-)$ & $-(-)$ & B \\
\hline Providencia rettgeri ATCC $29944^{\mathrm{T}}$ & 27 & + & - & - & ND & $100(27)$ & $+(+)$ & $-(-)$ & $-(-)$ & B \\
\hline Providencia rustigianii ATCC $33673^{\mathrm{T}}$ & 27 & + & - & - & ND & $100(27)$ & $+(+)$ & $-(-)$ & $-(-)$ & B \\
\hline Providencia stuartii ATCC $29914^{\mathrm{T}}$ & 25 & - & + & + & + & 25 & - & + & + & A1 \\
\hline & 27 & + & - & - & ND & $100(27)$ & $+(+)$ & $-(-)$ & $-(-)$ & B \\
\hline $\begin{array}{l}\text { Salmonella enterica serovar typhi } \\
\text { ATCC } 19430^{\mathrm{T}}\end{array}$ & 27 & + & - & - & ND & $100(27)$ & $+(+)$ & $-(-)$ & $-(-)$ & B \\
\hline \multirow{2}{*}{$\begin{array}{l}\text { Salmonella enterica serovar typhimurium } \\
\quad \text { ATCC } 15277\end{array}$} & 28 & - & + & + & - & 28 & - & + & + & A2 \\
\hline & 27 & + & - & - & ND & $100(27)$ & $+(+)$ & $-(-)$ & $-(-)$ & B \\
\hline Serratia plymuthica CIP 7712 & 28 & - & + & + & - & 28 & - & + & + & A2 \\
\hline Shigella dysenteriae NCTC $4837^{(\mathrm{T})}$ & 27 & + & - & - & ND & $100(27)$ & $+(+)$ & $-(-)$ & $-(-)$ & B \\
\hline Shigella flexneri CIP 8248 & 27 & + & - & - & ND & $100(27)$ & $+(+)$ & $-(-)$ & $-(-)$ & B \\
\hline Yokenella regensburgei ATCC 35313 & 25 & - & + & + & + & 25 & - & + & + & A1 \\
\hline
\end{tabular}

${ }^{a}$ See Materials and Methods for details concerning the assay procedures used.

${ }^{b} \mathrm{ND}$, not determined.

c The data in parentheses are data for the small amount of class B enzyme that was completely denatured under the conditions which we used (see Fig. 1).

has been variously involved in mutations, rearrangements, or even lateral exchange events within the family, which eventually caused the heterogeneous expression pattern observed now.

The production of class A Lmmp-APs was also found to be quite widespread among enteric bacteria, and most of the class A enzymes were class A1 enzymes. In fact, in addition to the Morganella morganii enzyme, class A1 Lmmp-APs were found in strains belonging to Cedecea davisae, Cedecea neteri, Enter- obacter aerogenes, Hafnia alvei, Klebsiella oxytoca, Klebsiella planticola, Klebsiella pneumoniae, Klebsiella terrigena, Providencia stuartii, and Yokenella regensburgei, while a class A2 enzyme apparently similar to the Salmonella enterica serovar typhimurium enzyme was found only in a strain of Serratia plymuthica. On the basis of these data and current knowledge concerning enterobacterial evolution $(1,11)$, we speculated that the class A1 enzymes have a phylogenetic history similar to that hypothesized for class B Lmmp-APs (see above), a view 
which is consistent with sequencing data which suggest that the class A1 Lmmp-APs of Morganella morganii and Providencia stuartii were acquired early in the lineage (21). On the other hand, the presence of only two class A2 enzymes, one of which (the PhoN enzyme of Salmonella enterica serovar typhimurium) is known to have been acquired recently by horizontal transfer (4), suggests that the class A2 Lmmp-AP found in Serratia plymuthica CIP 7712 may have a similar origin and that the class A2 Lmmp-APs found in enteric bacteria are derived from species other than members of the Enterobacteriaceae. Our findings, therefore, are consistent with the view that class A1 and A2 Lmmp-APs have different phylogenies. It would be interesting to investigate the relationship between the class A2 enzymes found in Serratia plymuthica CIP 7712 and Salmonella enterica serovar typhimurium ATCC 15277 (= LT2).

It should be noted, however, that investigation of a single strain may not be sufficient to account for the diversity of a whole species. It will be interesting to examine several strains of each species for production of Lmmp-APs to confirm that our results are actually characteristic for the various species.

\section{ACKNOWLEDGMENTS}

This work was supported in part by grants 93.1030.PF70 (targeted project "Biotecnologie e Biostrumentazione"), 93.04635.CT04, 94. 02875.CT04, and 94.02925.CT04 from the Italian National Research Council.

We thank Francesco Lissi and Elena Sestini for skillful secretarial assistance.

\section{REFERENCES}

1. Ahmad, S., W. G. Weisburg, and R. A. Jensen. 1990. Evolution of aromatic amino acid biosynthesis and application to the fine-tuned phylogenetic positioning of enteric bacteria. J. Bacteriol. 172:1051-1061.

2. Cocks, G. T., and A. C. Wilson. 1972. Enzyme evolution in the Enterobacteriaceae. J. Bacteriol. 110:793-802.

3. Edwards, C. J., D. J. Innes, D. M. Burns, and I. F. Beacham. 1993. UDPsugar hydrolase isozymes in Salmonella enterica and Escherichia coli: silent alleles of $u s h A$ in related strains of group I Salmonella isolates, and of $u s h B$ in wild-type and K12 strains of $E$. coli, indicate recent and early silencing events, respectively. FEMS Microbiol. Lett. 114:293-298.

4. Groisman, E. A., M. H. Saier, Jr., and H. Ochman. 1992. Horizontal transfer of a phosphatase gene as evidence for mosaic structure of the Salmonella genome. EMBO J. 11:1309-1316.

5. Izard, D., F. Gavini, P. A. Trinel, F. Krubwa, and H. Leclerc. 1980. Contribution of DNA-DNA hybridization to the transfer of Enterobacter aerogenes to the genus Klebsiella as $\mathrm{K}$. mobilis. Zentralbl. Bakteriol. Parasitenkd. Infektionskr. Hyg. Abt. 1 Orig. Reihe C 1:257-263.

6. Kasahara, M., A. Nakata, and H. Shinagawa. 1991. Molecular analysis of the Salmonella typhimurium phoN gene, which encodes nonspecific acid phos- phatase. J. Bacteriol. 173:6770-6775

7. Kier, L. D., R. Weppelman, and B. N. Ames. 1977. Resolution and purification of three periplasmic phosphatases of Salmonella typhimurium. J. Bacteriol. 130:399-410.

8. Laemmli, U. K. 1970 . Cleavage of structural proteins during the assembly of the head of bacteriophage T4. Nature (London) 227:680-685.

9. Lawrence, J. G., H. Ochman, and D. L. Hartl. 1991. Molecular and evolutionary relationships among enteric bacteria. J. Gen. Microbiol. 137:19111921.

10. Neu, H. C. 1968 . The $5^{\prime}$-nucleotidases and cyclic phosphodiesterases ( $3^{\prime}$ nucleotidases) of the Enterobacteriaceae. J. Bacteriol. 95:1732-1737.

11. Ochman, H., and A. C. Wilson. 1987. Evolutionary history of enteric bacteria, p. 1649-1654. In F. C. Neidhardt, J. L. Ingraham, K. B. Low, B. Magasanik, M. Schaechter, and H. E. Umbarger (ed.), Escherichia coli and Salmonella typhimurium. Cellular and molecular biology. American Society for Microbiology, Washington, D. C.

12. Pompei, R., G. Cornaglia, A. Ingianni, and G. Satta. 1990. Use of a novel phosphatase test for simplified identification of species of the tribe Proteeae. J. Clin. Microbiol. 28:1214-1218.

13. Pompei, R., A. Ingianni, G. Foddis, G. Di Pietro, and G. Satta. 1993. Patterns of phosphatase activity among enterobacterial species. Int. J. Syst. Bacteriol. 43:174-178.

14. Pradel, E., and P. L. Boquet. 1988. Acid phosphatases of Escherichia coli: molecular cloning and analysis of agp, the structural gene for a periplasmic acid glucose phosphatase. J. Bacteriol. 170:4916-4923.

15. Riccio, M. L., G. M. Rossolini, G. Lombardi, A. Chiesurin, and G. Satta. Unpublished data.

16. Rossolini, G. M., M. C. Thaller, R. Pezzi, and G. Satta. 1994. Identification of an Escherichia coli periplasmic acid phosphatase containing a $27-\mathrm{kDa}$ polypeptide component. FEMS Microbiol. Lett. 118:167-174.

17. Sambrook, J., E. F. Fritsch, and T. Maniatis. 1989. Molecular cloning: a laboratory manual, 2nd ed. Cold Spring Harbor Laboratory, Cold Spring Harbor, N. Y.

18. Satta, G., R. Pompei, G. Grazi, and G. Cornaglia. 1988. Phosphatase activity is a constant feature of all isolates of all major species of the family Enterobacteriaceae. J. Clin. Microbiol. 26:2637-2641.

19. Schlesinger, M. J., and R. Olsen. 1968. Expression and localization of Escherichia coli alkaline phosphatase synthesized in Salmonella typhimurium cytoplasm. J. Bacteriol. 96:1601-1605.

20. Thaller, M. C. Unpublished data.

21. Thaller, M. C., F. Berlutti, S. Schippa, G. Lombardi, and G. M. Rossolini 1994. Characterization and sequence of PhoC, the principal phosphateirrepressible acid phosphatase of Morganella morganii. Microbiology 140 $1341-1350$.

22. Thaller, M. C., G. Lombardi, F. Berlutti, S. Schippa, and G. M. Rossolini. 1995. Cloning and characterization of the NapA acid phosphatase/phosphotransferase of Morganella morganii: identification of a new family of bacterial acid phosphatase-encoding genes. Microbiology 141:147-154.

23. Uerkvitz, W. 1988. Periplasmic nonspecific acid phosphatase II from Salmonella typhimurium LT2. J. Biol. Chem. 263:15823-15830.

24. Uerkvitz, W., and C. F. Beck. 1981. Periplasmic phosphatases in Salmonello typhimurium LT2. A biochemical, physiological, and partial genetic analysis of three nucleoside monophosphate dephosphorylating enzymes. J. Biol. Chem. 256:382-389.

25. Weppelman, R., L. D. Kier, and B. N. Ames. 1977. Properties of two phosphatases and a cyclic phosphodiesterase of Salmonella typhimurium. J. Bacteriol. 130:411-419. 